\title{
Coupled optical defect microcavities in 1D photonic crystals and quasi-normal modes
}

\author{
M. Maksimovic, M. Hammer, E. van Groesen \\ MESA $^{+}$Institute for Nanotechnology, University of Twente, The Netherlands
}

\begin{abstract}
We analyze coupled optical defect cavities realized in finite one-dimensional Photonic Crystals. Viewing these as open systems where waves are permitted to leave the structures, one obtains eigenvalue problems for complex frequencies (eigenvalues) and Quasi-Normal-Modes (eigenfunctions). Single defect structures (photonic crystal atoms) can be viewed as elementary building blocks for multiple-defect structures (photonic crystal molecules) with more complex functionality. The QNM description links the resonant behavior of individual PC atoms to the properties of the PC molecules via eigenfrequency splitting. A variational principle for QNMs permits to predict the eigenfield and the complex eigenvalues in PC molecules starting with a field template incorporating the relevant QNMs of the PC atoms. Further, both the field representation and the resonant spectral transmission close to these resonances are obtained from a variational formulation of the transmittance problem using a template with the most relevant QNMs. The method applies to both symmetric and nonsymmetric single and multiple cavity structures with weak or strong coupling between the defects.
\end{abstract}

\section{INTRODUCTION}

Photonic Crystal (PC) based devices attracted much interest in the past two decades concerning both fundamental and applied aspects. Plenty of modeling and computational techniques are applied and well established. ${ }^{1-3}$ We consider 1-D PC structures that can provide qualitative insight and means for interpreting the physics of higher dimensional structures. More specifically, we consider planar layered inhomogeneous media with piecewise constant refractive index as the traditional model of 1-D PCs. Although they belong to the field of multilayer optics, ${ }^{4}$ an old and well explored field, a novel way of modeling these devices has certain theoretical and practical interest for itself.

The open and finite nature of realistic structures is accessible by directly characterizing resonance properties via an investigation of the quasi-normal modes and associated complex frequencies. Quasi-normal modes (QNMs) are eigenfunctions associated with the complex eigenfrequencies arising from the eigenvalue problem for outgoing waves. ${ }^{5}$ The real parts of the complex eigenfrequencies are connected with the transmission resonance frequencies (local maxima of the transmission) and the imaginary parts with the Q-factors (or linewidth) of the resonant transmission profile. Properties of the QNMs and related PC structures have been addressed for 1-D PC structures in, ${ }^{6-8}$ while for 2-D PC structures the theory is by far less often addressed and developed, with only partial results. ${ }^{9}$

We specialize to finite PC structures with suitable defects in otherwise periodic arrangements. These defects are forming Fabry-Perot cavities enclosed by and separated by leaky mirrors that allow the exchange of energy between cavities. These Coupled Optical Microcavites (CMC) already attracted research interest as they provide means for the implementation of optical filters, resonators, delay lines and other devices in both passive and active structures. ${ }^{3,10-13}$ Reference method for analyzing one-dimensional structures is a Transfer Matrix Method (TMM). ${ }^{4}$ A description in the framework of different coupled mode theory approaches has been a traditional way of analysis, ${ }^{14-17}$ as far as interacting optical waveguides (i.e., mostly systems with well confined optical states) are concerned. However, an analysis of open, leaky structures directly based on QNMs seems to be missing. This paper considers some possibilities for the direct characterization of open cavities in 1-D PC structures using only the most relevant QNMs.

Composite CMC structures can be viewed as being formed from simpler single cavity structures or some other elementary building blocks. This decomposition is usually quite arbitrary and can be done in many different ways for a given structure. However, when the individual modes are well localized in the vicinity of their respective cavities, a field template for the composite structure can be based on the superposition of the individual cavity modes. In literature the basic structures are sometimes called "photonic crystal atoms" which are the elementary building blocks for more complex "photonic crystal molecules". The key idea is that by combining PC atoms with known properties more complex PC molecules can be obtained with engineered properties. Based on QNMs and a variational principle, our procedure enables the derivation 
of the properties of the composite structures in a constructive way using the known properties of the building blocks and certain design rules for the composite structure.

In the context of CMCs, we address the splitting of eigenfrequencies by using a variational principle together with the related QNMs of the individual cavities. QNMs of the composite structure (super-modes) can be approximated by this approach. Further, we use the characterization of the CMCs in terms of quasi-normal modes to describe approximately the resonant response to an external excitation in the frequency domain and the related field profiles. The approximate frequency domain description follows from a suitable variational formulation ${ }^{18}$ for the transmission problem, using the most relevant QNMs in establishing appropriate field templates. ${ }^{19}$

\section{THEORY}

We consider 1-D optical structures in the frequency domain under external excitation. The optical field $E(x)$ excited by the external influx $E_{i n c}=A_{i n c} e^{i\left(n_{i n} \omega / c\right) x}$, with $\omega \in \mathbb{R}$ and $A_{i n c}$ given, for vacuum speed of light $c$, satisfies the Helmholtz equation

$$
\partial_{x}^{2} E+\frac{\omega^{2}}{c^{2}} n^{2}(x) E=0
$$

on an interval $x \in[L, R]$, and transparent influx boundary conditions

$$
\left(\partial_{x} E+i \frac{\omega}{c} n_{i n} E\right)_{x=L}=2 i \frac{\omega}{c} n_{i n} A_{\text {inc }}, \quad\left(\partial_{x} E-i \frac{\omega}{c} n_{\text {out }} E\right)_{x=R}=0
$$

at the boundaries $x=L, R$. The exterior regions $x<L$ and $x>R$ are assumed to be homogeneous with refractive indices $n_{\text {in }}$ and $n_{\text {out }}$, respectively. For structures with piecewise constant refractive index an exact solution can be obtained via a standard and well known transfer matrix method; ${ }^{4}$ a brief explanation is given in appendix A. This serves as reference for the approximate models discussed below.

Properties of passive, open optical structures with energy exchange between the constitutive elements and the environment are captured adequately by a formulation of an eigenvalue problem for complex frequencies. A finite structure can be viewed as an open system with transparent boundaries which permit the leakage of energy to the exterior, see Figure 1 A). The electric field in the interior $x \in(L, R)$ satisfies the Helmholtz equation:

$$
\partial_{x}^{2} Q+\frac{\omega^{2}}{c^{2}} n^{2}(x) Q=0
$$

with outgoing wave boundary conditions

$$
\left(\partial_{x} Q+i \frac{\omega}{c} n_{i n} Q\right)_{x=L}=0, \text { and }\left(\partial_{x} Q-i \frac{\omega}{c} n_{\text {out }} Q\right)_{x=R}=0 .
$$

This constitutes an eigenvalue problem for the frequency $\omega$ as the complex eigenvalue and the field profile $Q(x)$ as eigenfunction (Quasi-Normal Mode). ${ }^{5-7,20}$ The eigenvalue problem is nonlinear because the eigenvalue appears in the boundary conditions explicitly. ${ }^{20}$ QNMs can be used to solve the initial-value problem of energy leakage out of a given open structure. The applicability of QNMs for solutions of the transmission problems with given influx relies on specific pseudoorthogonality and completeness properties of QNMs when used as a basis set for an eigenfunction expansion. 6,7

A variational formulation of the QNM eigenvalue problem can be based on the functional ${ }^{18}$

$$
\mathcal{L}_{\omega}(Q)=\frac{1}{2} \int_{L}^{R}\left(\left(\partial_{x} Q\right)^{2}-\frac{\omega^{2}}{c^{2}} n^{2}(x) Q^{2}\right) d x-\frac{i \omega}{2 c}\left(\left.n_{\text {in }} Q^{2}\right|_{x=L}+\left.n_{\text {out }} Q^{2}\right|_{x=R}\right) .
$$

If $\mathcal{L}_{\omega}$ becomes stationary, i.e. if the first variation of $\mathcal{L}_{\omega}(Q)$ vanishes for arbitrary variations of $Q$, then $Q$ satisfies equation (3) with equations (4) as natural boundary conditions. The value of the functional (5) with the proper eigenfunction/ eigenvalue pair $(\omega, Q)$ inserted is zero, i.e.

$$
\mathcal{L}_{\omega}(Q)=0 \text {. }
$$

This property can be shown analytically by computing the partial derivative of the first term in the (5).

We specialize to the analysis of optical defect modes existing in the bandgap of the underlying periodic structure. To avoid using the full set of QNMs and the completeness properties of QNMs to determine approximations of the optical transmission and of the related field profiles, we apply a variational principle and a specific field template that consists of QNMs associated only with the optical defects. Details of this procedure can be found in ${ }^{19}$ and in appendix B. 


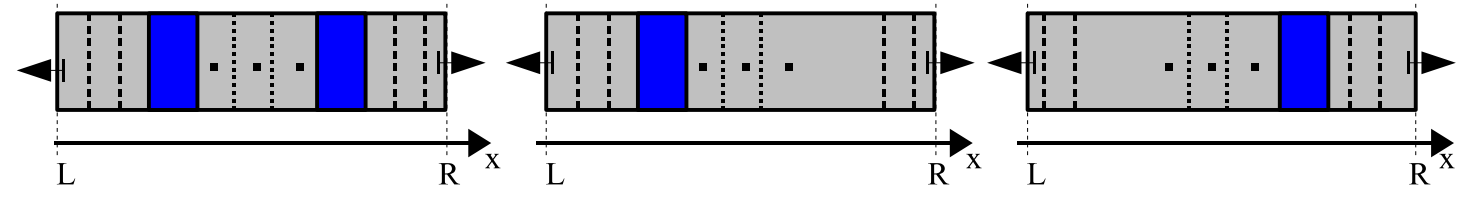

Figure 1. The coupled optical defect structures considered in this paper are finite periodic multilayer structures consisting of two materials with high index $n_{H}$ and low index $n_{L}$. The layer thicknesses $d_{H}, d_{L}$ are chosen to be quarter-wavelength for the target wavelength (related to a reference frequency $\omega_{0}$ ). Optical defects are introduced as changes of the layer thicknesses or refractive indices in the otherwise periodic sequence. The whole structure is enclosed by two semi-infinite media of indices $n_{\text {in }}$ and $n_{\text {out }}$. A composite multiple defect structure A) can be decomposed into usually simpler single defect structures $B$ ) and C).

\subsection{Coupled cavities}

We start with the QNMs $\left(\omega_{1}, Q_{1}\right), \ldots,\left(\omega_{N}, Q_{N}\right)$ for refractive index distributions $n_{1}(x), \ldots, n_{N}(x)$ of simpler (not necessarily single cavity) structures. Solutions of the eigenvalue problem for the composite structure are assumed to be well approximated by linear combinations of the QNMs belonging to the simpler structures. Therefore, we choose the field template

$$
Q=\sum_{p=1}^{N} a_{p} Q_{p}
$$

which represents a restriction $\mathcal{L}_{\omega}(Q) \rightarrow L_{\omega}\left(a_{1}, \ldots, a_{N}\right)$ of the solutions of the original problem. Stationarity of the functional (5) transforms on the restricted set to the conditions

$$
\frac{\partial L_{\omega}}{\partial a_{p}}\left(a_{1}, \ldots, a_{p}, \ldots, a_{N}\right)=0, \text { for } p=1, \ldots, N,
$$

that can be written as an algebraic quadratic eigenvalue problem $^{21}$

$$
\left(\omega^{2} \mathbf{M}+\omega \mathbf{N}+\mathbf{P}\right) \mathbf{a}=0
$$

for the complex eigenfrequencies $\omega$ of the composite system. The eigenvectors $\mathbf{a}=\left[a_{1}, \ldots, a_{N}\right]^{T}$ are the unknown coefficients in the linear superposition (7) of the single cavity QNMs. The elements of the matrices $\mathbf{M}=\left[M_{l k}\right]_{N \times N}$, $\mathbf{N}=\left[N_{l k}\right]_{N \times N}, \mathbf{P}=\left[P_{l k}\right]_{N \times N}$ are

$$
M_{l k}=-\frac{1}{c^{2}} \int_{L}^{R} n^{2}(x) Q_{l} Q_{k} d x, \quad N_{l k}=-\frac{i}{c}\left(\left.n_{\text {in }} Q_{l} Q_{k}\right|_{x=L}+\left.n_{\text {out }} Q_{l} Q_{k}\right|_{x=R}\right), \quad P_{l k}=\int_{L}^{R} \partial_{x} Q_{l} \partial_{x} Q_{k} d x .
$$

Equation (9) enables the approximate solution of the eigenvalue problem for the composite structure. It directly links the resonance behavior of the individual constitutive elements (PC atoms) to the resonance properties of more complex structures (PC molecules), i.e. describes the eigenfrequency splitting. Both resonant frequencies and the related Q-factors can be estimated. Influences of the external and internal confinement (type, length and strength of the "mirrors" in the structure) or perturbations of various parameters can be directly analyzed.

Usually the decompositions of the composite structure, i.e. the precise choice of the elements $Q_{p}$ in (7) is to some degree arbitrary. Supporting arguments can be based on results from direct computations, on physical intuition, but also on the following observation. For fields $Q_{l}$ with associated frequency $\omega_{l}$ and refractive index $n_{l}$ that satisfy (3), (4), equation (9) can be written as

$$
\mathbf{S a}=0,
$$

where

$$
S_{l k}=\int_{L}^{R} \frac{\omega^{2} n^{2}(x)-\omega_{l}^{2} n_{l}^{2}(x)}{c^{2}} Q_{l} Q_{k} d x+\frac{i\left(\omega-\omega_{l}\right)}{c}\left(\left.n_{\text {in }} Q_{l} Q_{k}\right|_{x=L}+\left.n_{\text {out }} Q_{l} Q_{k}\right|_{x=R}\right) .
$$

If the trial field includes the exact solution for the composite structure with the property $\omega=\omega_{l}$ then (11) is satisfied. Expression (12) suggests that the refractive index distributions $n_{l}$ of the simpler structures in the decomposition should be chosen as close as possible to the exact structure (refractive index $n$ ). 


\subsection{First order perturbation correction for complex eigenfrequencies}

We look for corrections of the complex eigenfrequencies for a given structure when small, localized perturbations of the permittivity are present. A first order perturbation correction for the complex eigenvalue can be obtained by using (5) and a known QNM eigenpair $\left(\omega_{0}, Q_{0}\right)$ of the unperturbed problem with refractive index distribution $n_{0}(x)$. It is reasonable to assume that a small perturbation of the original structure does neither change substantiality the position of the complex eigenfrequencies in the complex plane nor the shape of the corresponding QNMs. We consider a permittivity perturbation in the form

$$
n^{2}(x)=n_{0}^{2}(x)+n_{p}^{2}(x) .
$$

For small (in effect) perturbations $n_{p}^{2}$ we look for a first order correction $\omega_{1}$ to the eigenfrequency $\omega=\omega_{0}+\omega_{1}$. Variational accuracy guarantees that the eigenfrequency is determined up to first order if the eigenfunction is known up to zeroth order (solution of the unperturbed structure). Upon restricting (5) to the zeroth order field approximation $\mathcal{L}_{\omega}\left(a Q_{0}\right) \rightarrow L(a)$, the stationarity condition on the restricted set

$$
\frac{\partial L}{\partial a}(a)=0
$$

gives an equation for the eigenfrequency correction. Keeping only the first order terms in $\omega_{1}$ and using the property (6) satisfied by the eigenpair $\left(\omega_{0}, Q_{0}\right)$ of the unperturbed problem, the correction to the complex eigenfrequency reads

$$
\omega_{1}=-\frac{\omega_{0}^{2}}{c^{2}} \frac{\int_{L}^{R} n_{p}^{2}(x) Q_{0}^{2} d x}{2 \frac{\omega_{0}}{c^{2}} \int_{L}^{R} n_{0}^{2}(x) Q_{0}^{2} d x+\frac{i}{c}\left(\left.n_{\text {in }} Q_{0}^{2}\right|_{x=L}+\left.n_{\text {out }} Q_{0}^{2}\right|_{x=R}\right)} .
$$

Obviously this procedure is closely related to the theory of (2.1); it may be viewed as a "coupled mode theory" with only one mode in the template (7). It is possible to extend this method and to derive both corrections to the eigenvalue and to the eigenfunction up to arbitrary order using a variational principle. An iterative procedure for higher order corrections will be reported elsewhere.

\section{RESULTS AND DISCUSSION}

A series of examples of CMCs serves to validate the described methods. First, we apply the variational principle of Section 2.1 for approximating supermodes in a double-cavity structure using known QNMs of the individual single cavities. Second, the variational form of first order perturbation theory for QNMs (Section 2.2) is used to analyze shifts of cavity resonances subjected to local perturbations of the refractive index. Third, the method of appendix B is applied to estimate the transmission on the basis of a few, most relevant QNMs. Finally, we consider multiple-defect structures designed to operate in weak and in strong coupling regimes. Also here our variational approximation method links the resonant transmission to the underlying QNMs.

\subsection{Double cavity structure}

Consider a layer arrangement coded as $(H L)^{M_{1}} D(L H)^{M_{1}}$, where $M_{1}=4$ is the number of layer pairs in two mirrors that enclose a single cavity, with $n_{H}=3.42, n_{L}=1.0$, between two semi-infinite media of the same refractive index $n_{\text {in }}=n_{\text {out }}=1.0$. The defect is introduced as a central layer of thickness $d_{D}=2 d_{H}$ with high refractive index $n_{D}=n_{H}$. A complex QNM eigenfrequency associated with the defect is present in the bandgap region of the related periodic structure. This eigenfrequency has an imaginary part that is several orders of magnitude smaller (absolute value) than all other eigenfrequencies in the QNM spectrum. ${ }^{19}$ Usually this is a sign of a strong localization of the field, i.e. for efficient energy trapping in the vicinity of the defect.

The combination $(H L)^{M_{1}} D(L H)^{M_{2}} L D(L H)^{M_{1}}$ of two of these single cavity structures constitutes a multilayer arrangement with two defects and three mirrors (two enclosing mirrors of "length" $M_{1}$, one separating mirror of length $M_{2}$ ). The defects form two Fabry-Perot-like resonant cavities with two corresponding QNMs and eigenfrequencies, see Figure 2 A). These eigenfrequencies correspond to two transmission resonances (Figure 2 B) ). The resonant response of the double-cavity structure (the PC molecule) can be viewed as being generated through eigenfrequency splitting from the resonance of the single cavities (the PC atoms). By changing the number $M_{2}$ of pairs in the separating mirror one can 
control the interaction strength between the two cavities, where the relative distance of the complex frequencies reflects weak or strong coupling. If the separation is small, the overlap of the individual QNMs is substantial, which results in a strong separation of eigenfrequencies. Increasing the separation leads to close eigenfrequencies and results in the formation of a transmission pass-band. With a field template (7) that consists of a linear superposition of the two QNMs associated with the individual left and right cavities, the procedure of Section 2.1 permits the estimation of both eigenfrequencies and QNMs of the PC molecule. According to Figure 2 this is an excellent approximation even for quite moderate cavity separations $M_{2}$ with rather strong interaction.

In contrast to the composite structure, the permittivity profiles that constitute the PC atoms do not show a particular symmetry (cf. Figure 1). Hence the QNMs associated with the individual cavities do not exhibit a special symmetry. When the decomposition is performed properly, however, their symmetric and skew-symmetric linear combinations approximate the symmetric and skew-symmetric supermodes of the composite structure, see Figure 2 C) and D).

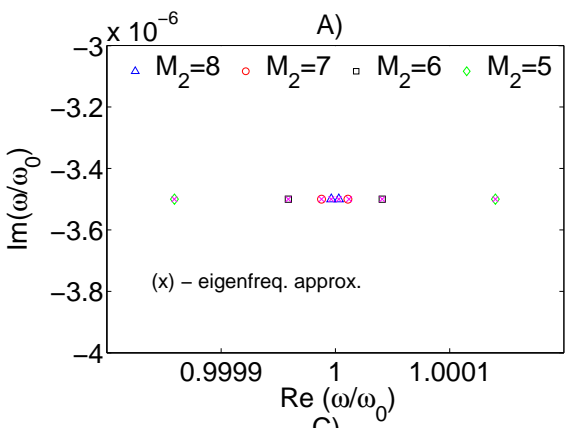

C)

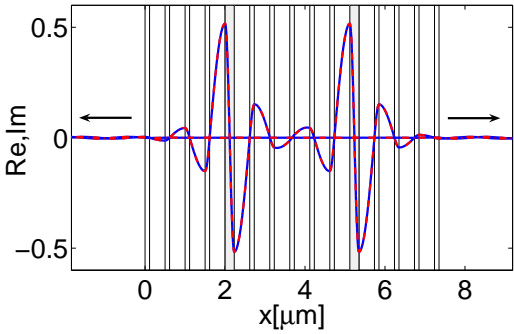

B)

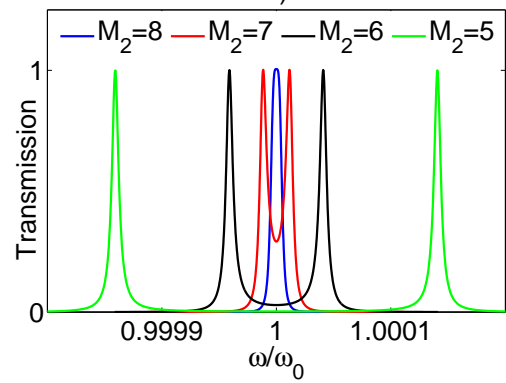

D)

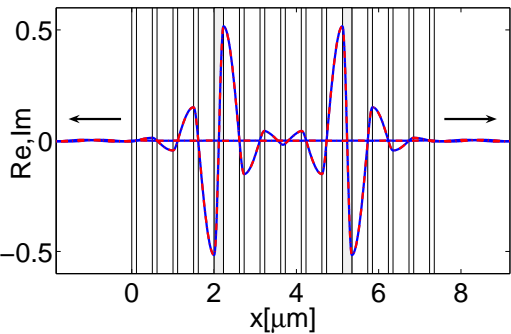

Figure 2. A): complex eigenfrequencies $\omega$ for the double cavity structure, direct computations and CMT approximations for different lengths $M_{2}$ of the separation region; $B)$ : transmittance, direct TMM calculation; C), D): QNMs (supermodes) for the double cavity structure with $M_{2}=5$, direct computation (continuous) and CMT approximation (dashed).

Further, the variational method of appendix B allows to characterize the contributions of individual QNMs to the spectral transmission. Figure 3 compares two different settings: The template (21) for the transmission field can be based either on the two (exact) supermodes of the PC molecule, or on the QNMs supported by the PC atoms. In both cases the resulting approximations for the transmission are indistinguishable (on the scale of the figure) from the TMM reference. Especially interesting is the weak coupling regime, where the direct computation based on the TMM method can not easily explain the resonant character of the transmission band. However, examination of the relevant complex eigenvalues, of the QNMs, and of the expansion coefficients describes completely the resonant character of the transmission band.

\subsubsection{Perturbation of the double cavity structure}

The perturbation theory from Section 2.2 is applied to analyze eigenfrequency shifts due to small local perturbations of the cavity refractive index. Below we look at both symmetric and asymmetric perturbations of the symmetric original structure. The perturbative correction for QNMs estimates reasonably, in first order, both real and imaginary parts of the complex eigenfrequencies. This can be traced further to changes of the transmission, i.e. to the position of resonance frequencies and the related Q-factors. Figure 4 introduces the specific configuration.

First we consider an asymmetric perturbation, where the refractive index of only one of the defects is raised locally. According to Figure 5, this leads not only to shifts in the positions of the eigenfrequencies (A)), but also to dramatic changes of the transmission response (B)). The perturbation corrections (15) are obtained here with the QNMs (supermodes) of the original composite structure. Figure $5 \mathrm{~A}$ ) shows the paths of the eigenfrequencies in the complex plane for varying strength $p$ of the perturbation, where the influence of the refractive index change has been evaluated by expression 
A)

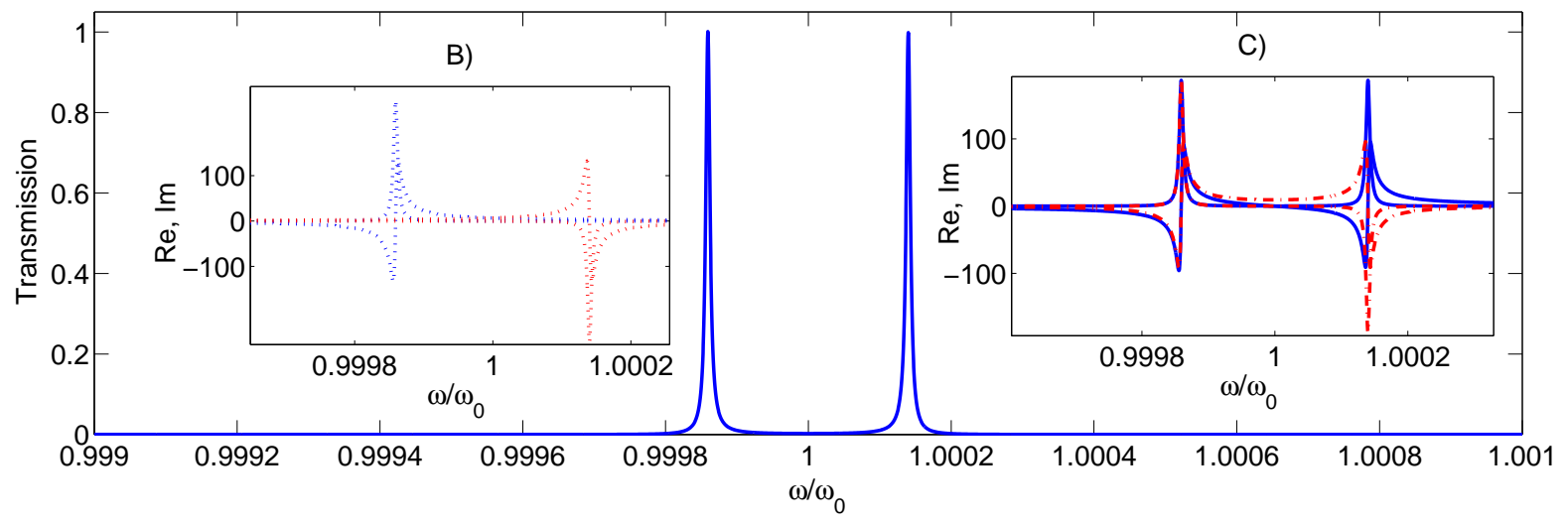

D)

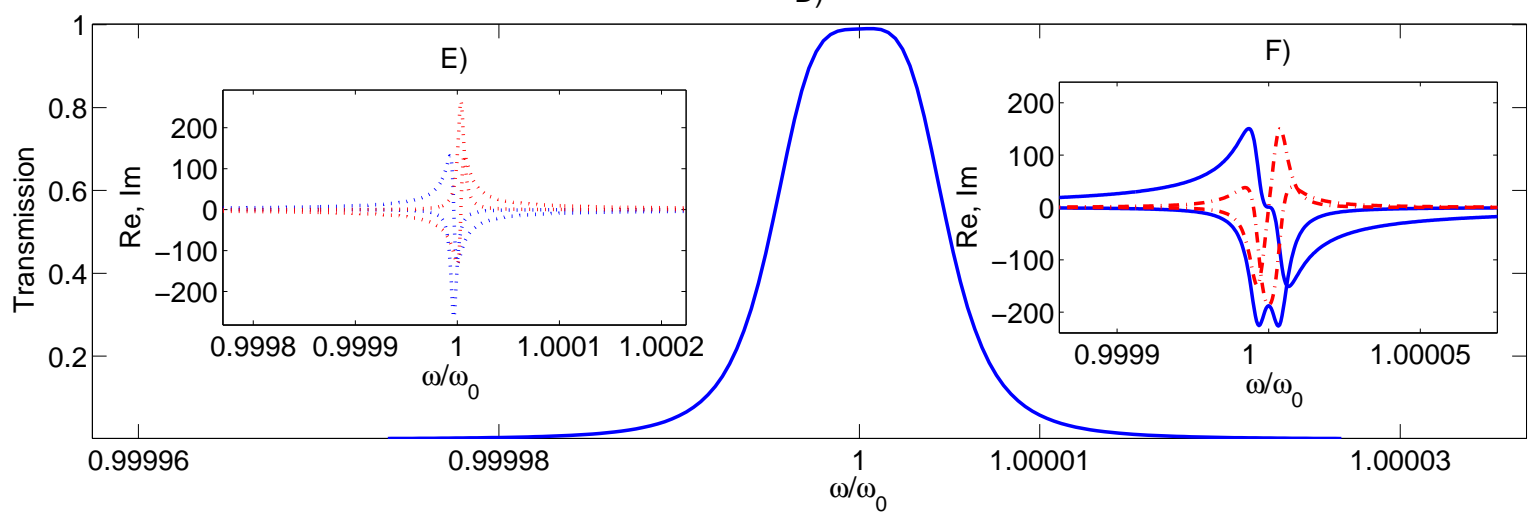

Figure 3. Transmittance (large axes, A), D); direct TMM computations and CMT model, superimposed curves) and coefficients $a_{p}$ for approximations (appendix B) to the transmission problem, if the template (21) includes either the two exact supermodes of the composite structure (left insets B), E)) or alternatively the QNMs associated with the individual left and right cavities (right insets $C$ ), F)). The upper plots $A), B$ ), C) correspond to a moderate cavity separation $M_{2}=5$, the lower plots $\left.D\right), E$ ), F) to a setting with $M_{2}=8$, i.e. with weaker interaction.

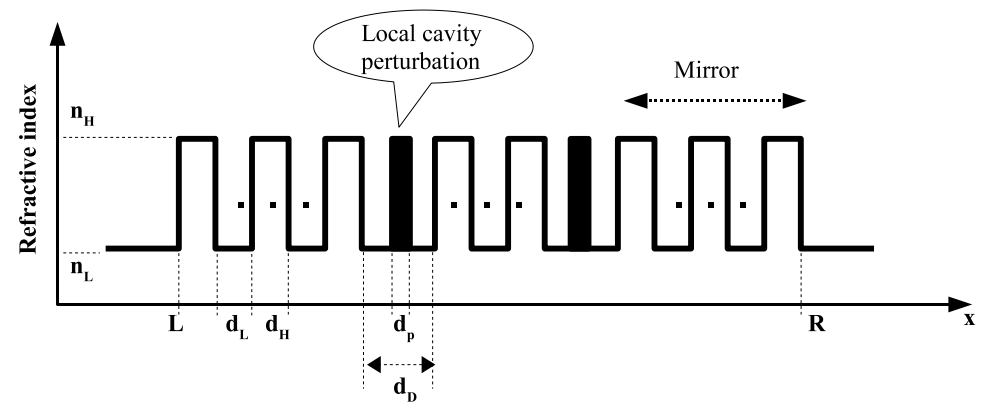

Figure 4. Perturbation of a CMC by localized refractive index changes. The double cavity structure is encoded as $(H L)^{4} H(2 L)(H L)^{8} H(2 L)(H L)^{4} H$. The individual layers with alternately high $(H)$ and low refractive index $(L)$ are quarter-wavelength with $n_{H}=1.5, n_{L}=1, n_{\text {in }}=n_{\text {out }}=1$. Two low index layers with larger half-wavelength thickness $d_{D}$ form the two defects. Perturbations are introduced as local changes of the permittivity $n^{2}=n_{L}^{2}(1+p)$ in the middle of the defect layer with a thickness of $d_{p}=d_{D} / 5$ and $p \in(0,0.05)$.

(15) on the one hand, and, for comparison, by direct TMM calculations on the other hand. As expected, the straight lines given by the first order perturbational expression are tangential to the reference paths. In this case the range of a reasonable approximation level is rather limited, because the perturbation destroys the overall symmetry of the structure.

If, in contrast, both cavities are perturbed in a symmetrical way, the results of the perturbational procedure are accurate over a much larger range of perturbation strengths, as seen in Figure $5 \mathrm{C}$ ). Now the eigenfunctions of the perturbed structured retain their symmetry, i.e. the assumption that the QNM of the original structure forms an acceptable approxima- 
tion to the perturbed configuration is apparently better justified. For both the symmetric and the asymmetric perturbation, the variational procedure of appendix B, in Figure 5 B) and D) applied with the supermodes of the perturbed composite structure in the template, gives accurate results for the spectral transmission through the double cavity structure.
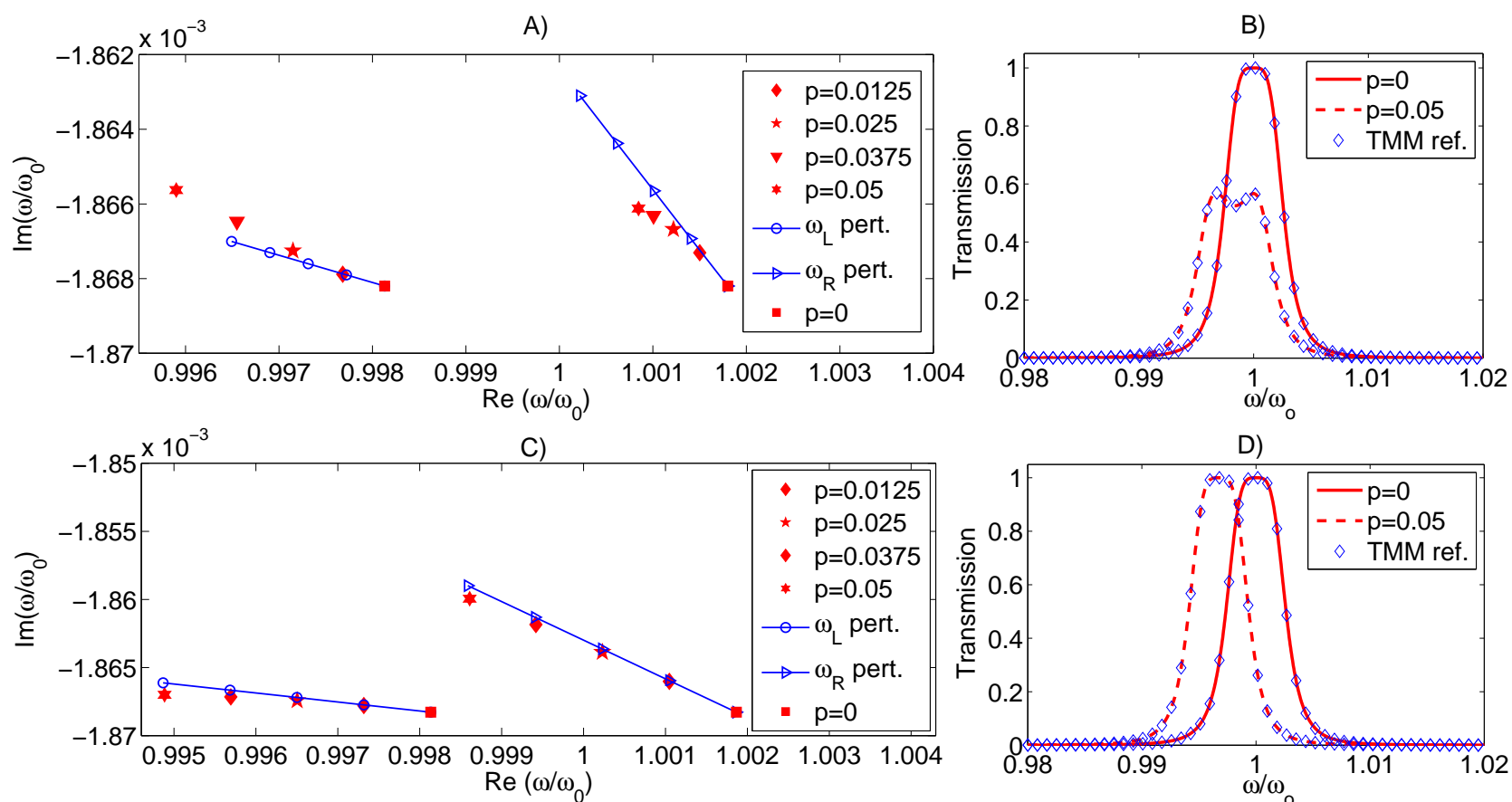

Figure 5. A), C): complex eigenfrequencies for the double cavity structure of Figure 4, direct computations and first order perturbation theory approximations; B), D): spectral transmittance, QNM approximation (appendix B) based on exact QNM supermodes, and TMM reference; asymmetric (A), B) ) and symmetric perturbations (C), D)).

For the asymmetrically changed double cavity configuration of Figure $5 \mathrm{~A}$ ) we observed that the perturbational expression (15) grossly over- or underestimated the QNM eigenvalue correction. This was attributed to the fact that the underlying field template could not respond to the broken symmetry of the perturbed structure. It is thus intriguing to try a modified template that combines separate QNMs of the two individual cavities, i.e. to apply the theory of Section 2.1. Necessarily with this procedure one encounters a certain error already for the approximation of the QNM supermode eigenfrequencies of the unperturbed, symmetric structure (observe that this concerns a configuration with relatively low refractive index contrast and strong interaction). Still, according to Figure 6 A), the eigenfrequency shifts predicted by the CMT formalism cover the whole range of perturbation strengths considered here with reasonable accuracy, at least as far as real parts are concerned. Plots B) and C) of Figure 6 show that the eigenfunctions of the perturbed structure are indeed not even approximately symmetric.

\subsection{Multiple cavity structures}

First, we look at the multiple cavity structure (the PC molecule) that is formed by repeating the former single cavity structure (the PC atom) according to the following design rule. Repetition of the unit cell $P C A_{1}=(H L)^{M_{1}}(2 H)(L H)^{M_{1}}$, here with $M_{1}=4$, generates the molecule $\left[P C A_{1}, L\right]_{J}$, where $J$ is the number of PC atoms. The refractive indices are the same as given in Figure 4 for the previous example. The plots A) and B) in Figure 7 show the complex frequencies and the resonant transmission for PC molecules with $J=2$ and $J=3$, respectively. Obviously these PC molecules operate in the weak coupling regime, as is reflected in the proximity of the eigenfrequencies (A) ) and in the characteristic transmission pass-band (B)). The transmission, estimated according to the recipes of appendix B with directly computed QNM supermodes of the molecule, is in the excellent agreement with the TMM reference. The number of relevant QNMs in the composite structure is equal to the number of $\mathrm{PC}$ atoms; modifications of this number permit a constructive tailoring of the transmission pass-band. For additional tuning of the transmission that might be of interest, such as ripple suppression 

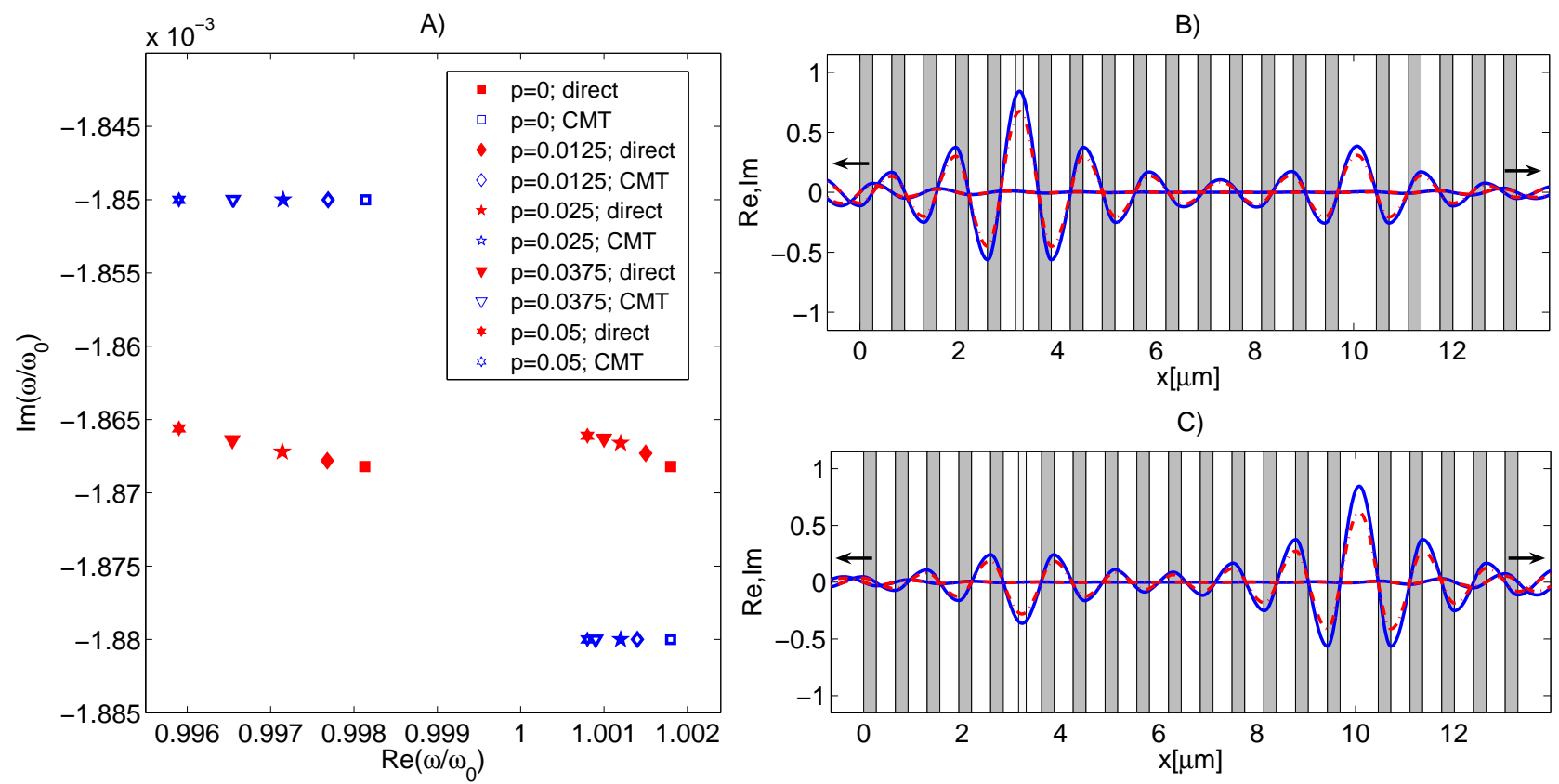

C)

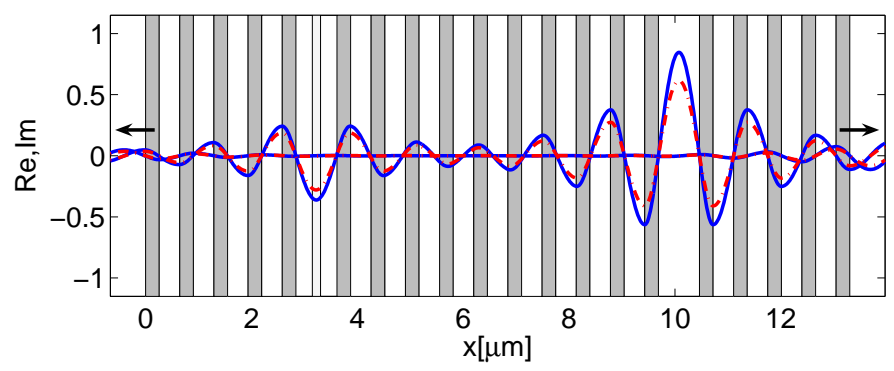

Figure 6. A): complex eigenfrequencies for the double cavity structure of Figure 4, direct TMM computations and CMT approximations, in the case of asymmetric perturbations. B), C), for or a perturbation strength $p=0.05$ : QNM profiles obtained with CMT (dashed lines) and direct computation (continuous).

(to optimize for a flat-top response), one could adjust the strength (number of layer pairs) of the mirrors, or add a certain degree of asymmetry to the final design. 3,19

Second, we consider the molecule formed by repeating the unit cell $P C A_{2}=(H L)^{M_{1}}(2 H)(L H)^{M_{2}} L(2 H)(L H)^{M_{1}}$, with $M_{1}=4$ and $M_{2}=2$ (a strongly coupled double cavity structure), coded as $\left[P C A_{2}, L\right]_{J}$. In Figure 7 the complex eigenfrequencies (C) ) and the spectral transmission (D) ) are shown. This procedure represents the design of a multiple channeled filter with narrow bandpass transmission. By proper adjustment of the inter cavity separation (i.e. of the coupling strength), the relative position of the transmission channels can be controlled. Additional unit cells contribute to the eigenfrequency splitting in such a way that the split eigenfrequencies are close. Therefore, no additional transmission bands appear but the width of the transmission pass-bands is narrowed.

Finally, a combination of the PC atoms $P C A_{1}$ and $P C A_{2}$ leads to an even more complex composite structure. The $\mathrm{PC}$ molecule is given by the sequence $P C M=\left[P C A_{1}, L, P C A_{2}, L, P C A_{1}\right]$. Figure 8 shows eigenfrequencies (A) ) and the corresponding QNMs (B)-E)). The individual contributions of each atom to the supermode profiles of the molecule are clearly visible. The eigenfrequencies $\omega_{2}$ and $\omega_{3}$ are the product of a weak coupling between the atoms $P C A_{1}$ (the single cavity structures), according to the shape of the corresponding QNMs (D), E)). The eigenfrequencies $\omega_{1}$ and $\omega_{4}$ originate from $P C A_{2}$ and are affected by $P C A_{1}$ only in the form of an increased confinement (i.e. a lower absolute value of the imaginary parts of the eigenfrequencies). The transmission for the composite structure exhibits a characteristic combination of both constitutive atoms. The high transmittance peaks are caused by the resonances associated with $P C A_{2}$, while the transmission resonances of $P C A_{1}$ are modulated (here they are suppressed) by the presence of $P C A_{2}$. In this case, light can not establish an efficient propagation path from the left $P C A_{1}$ to the right one, because the frequencies supported by $P C A_{1}$ are inside the attenuation region of $P C A_{2}$, see Figure 7.

We like to emphasize here that the QNM analysis can be very useful for an interpretation of results and for an accurate prediction of the outcome of transmission experiments, as shown in the previous paragraphs. Here, the approach establishes a sound foundation of the concept of photonic crystal molecules, that cannot be provided easily by direct TMM solutions. 

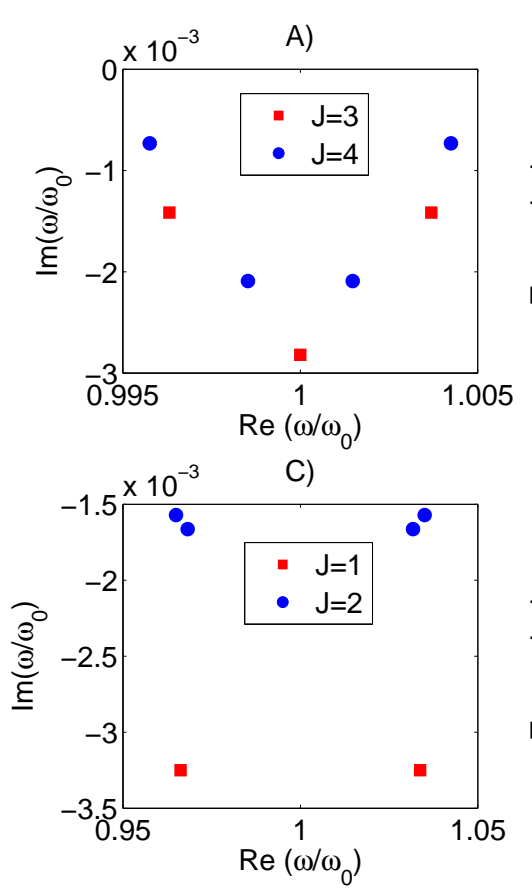

B)

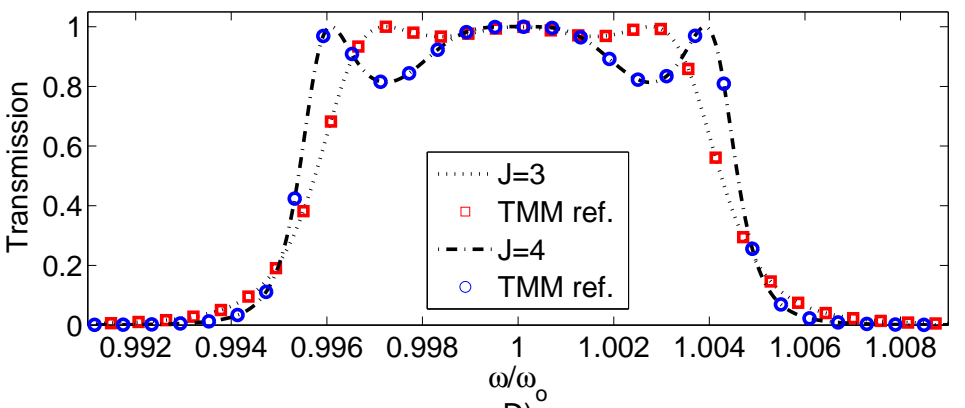

D) $)^{\circ}$

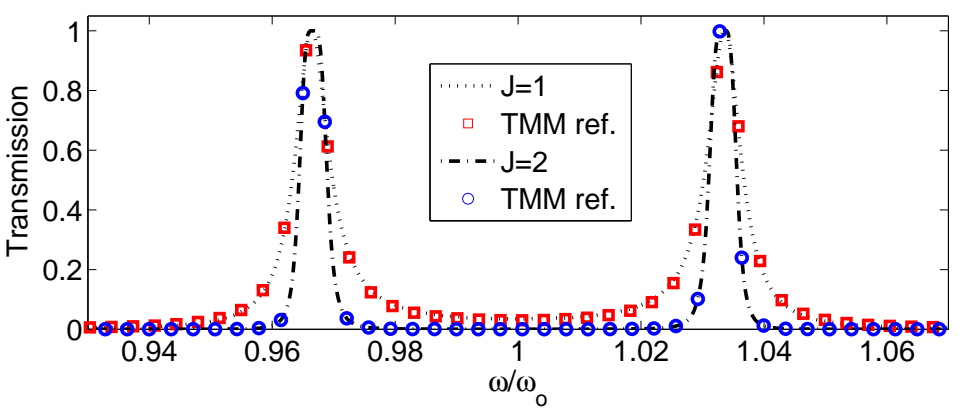

Figure 7. A), B): complex eigenfrequencies and transmission for weakly coupled multiple cavity structures $\left.\left[P C A_{1}, L\right]_{J} ; C\right), D$ ): frequencies and transmission for PC molecules $\left[P C A_{2}, L\right]_{J}$ formed by repeating a double cavity unit cell in the strong coupling regime.
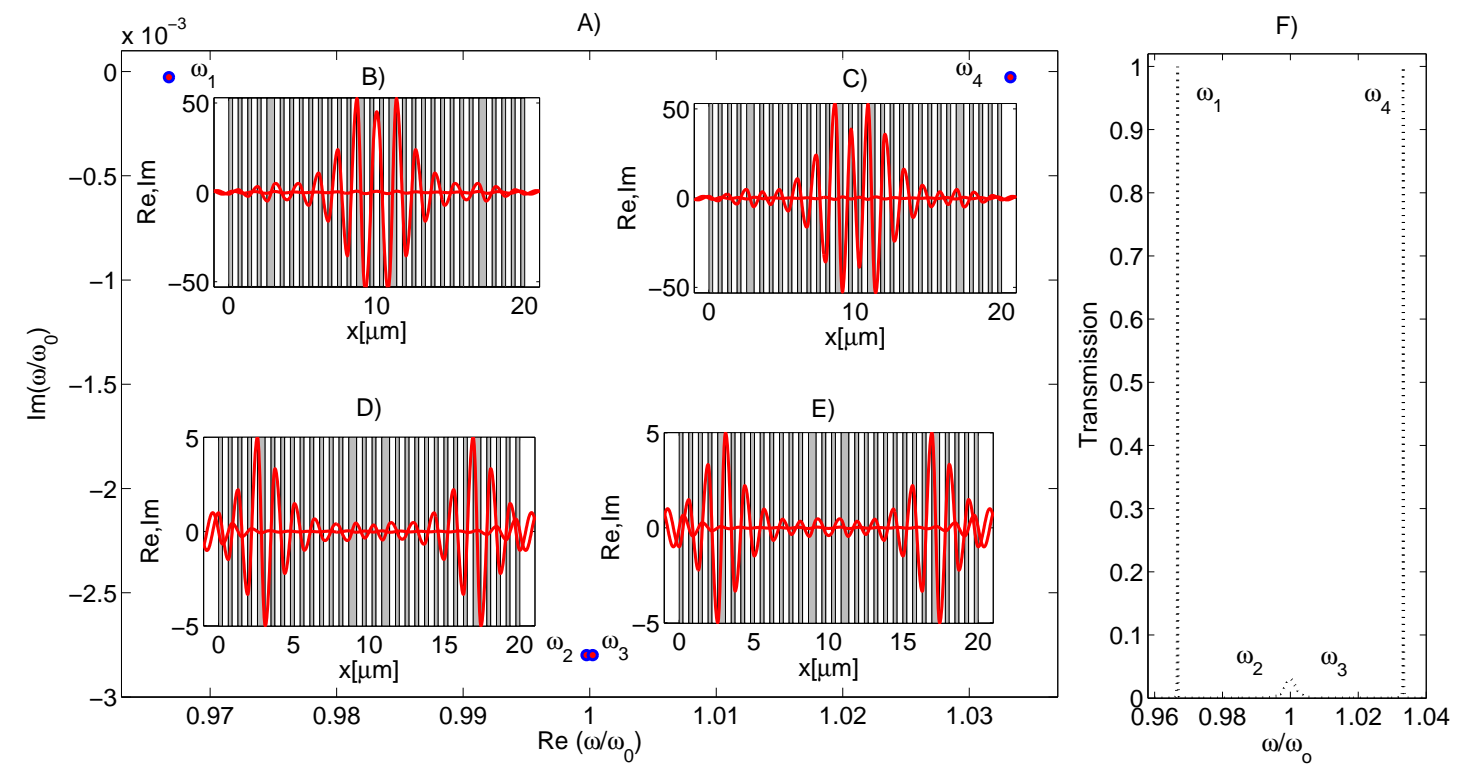

Figure 8. A): complex eigenfrequencies $\omega_{1}-\omega_{4}$ of the $P C$ molecule $\left[P C A_{1}, L, P C A_{2}, L, P C A_{1}\right]$ formed by combination of the single and double cavity atoms of Figure 7. The insets show the corresponding $Q N M s$, where $\omega_{1}, \omega_{2}, \omega_{3}$, and $\omega_{4}$ are related to profiles B), D), E), and C). F): spectral transmission for the composite structure.

\section{CONCLUSIONS}

In this paper we consider the open and finite nature of a specific class of PC structures by directly characterizing their resonance properties via an investigation of the quasi-normal mode spectrum. A variational principle for QNMs allows to approximate the eigenfrequencies and QNMs of composite multiple cavity structures by eigenfrequencies and QNMs 
of simpler structures. Further, a constructive, recently developed way ${ }^{19}$ of relating a quasi-normal mode description to transmission properties of optical defect microcavities in 1D PCs is applied. Detailed remarks about alternative existing methods can be found in. ${ }^{19}$

We specialize to defect structures that support transmission modes in the bandgap of otherwise periodical structures. Numerical examples show that the method is applicable for both symmetric and nonsymmetric layer arrangements and both weak and strong coupling between defects.

A form of coupled mode theory for finite, open 1-D PC structures is proposed, that uses directly the most relevant QNMs. Closely related, an expression for a first order perturbation correction of the complex eigenfrequencies is derived by means of variational restriction. In contrast to other methods that use different types of basis fields and rely either on a tight-binding approximation ${ }^{10,13}$ and/or on supercell methods, ${ }^{11,12}$ with our approach the finite nature of the individual building blocks in the composite structure is fully respected.

Further, we analyzed a series of characteristic examples of multiple cavity structures and were able to point out characteristic features in the composite structures as originating from simpler structures. The results suggest that the notion of the photonic crystal molecules can be founded on the QNM analysis as considered here. Together with our variational approximation method, the QNM analysis offers a resourceful method for the interpretation of complex phenomena associated with the resonance properties in 1-D PC structures.

Provided that suitable QNM basis fields can be made available by analytical or numerical means, possible generalizations to 2D and 3D structures could be based on suitable functional representations of the frequency domain Maxwell equations for higher dimensions. ${ }^{23}$

\section{APPENDIX A. TRANSFER MATRIX METHOD}

For structures with piecewise constant refractive index distribution inside a finite spatial domain a method for solving both the transmittance and eigenvalue problems is the well known transfer matrix method (TMM). ${ }^{4}$ Solutions of the Helmholtz equation are given as combinations of left- and right-traveling waves in the $j$-th layer

$$
E_{j}(x)=A_{j} e^{i k_{j}\left(x-l_{j-1}\right)}+B_{j} e^{-i k_{j}\left(x-l_{j-1}\right)}
$$

for $x \in\left[l_{j-1}, l_{j}\right]$ in a region of constant index $n_{j}$ where $k_{j}=n_{j} \omega / c$ is the wave number in this layer. To connect the fields inside all layers we impose continuity conditions at the interfaces:

$$
E_{j}\left(l_{j}\right)=E_{j+1}\left(l_{j}\right), \text { and } \partial_{x} E_{j}\left(l_{j}\right)=\partial_{x} E_{j+1}\left(l_{j}\right) .
$$

These conditions lead to a system of equations that can be represented in matrix form. Ordered multiplication of the relevant matrices connects amplitudes in each layer of the structure, as well as the amplitudes in the incidence and output regions:

$$
\left(\begin{array}{c}
A_{\text {in }} \\
B_{\text {in }}
\end{array}\right)=\left(\begin{array}{ll}
m_{11}(\omega) & m_{12}(\omega) \\
m_{21}(\omega) & m_{22}(\omega)
\end{array}\right)\left(\begin{array}{c}
A_{\text {out }} \\
B_{\text {out }}
\end{array}\right)
$$

The transmittance problem with incoming wave from the left is solved with $B_{\text {out }}=0$ for specified $A_{\text {in }}$ (amplitude of the incoming wave) with given real frequency $\omega \in R$. The amplitude transmission and reflection coefficients are expressed as

$$
t(\omega)=\frac{A_{\text {out }}}{A_{\text {in }}}, \text { and } r(\omega)=\frac{B_{\text {in }}}{A_{\text {in }}} .
$$

If we choose conditions $A_{\text {in }}=B_{\text {out }}=0$, i.e. restrict the exterior solutions to purely outgoing waves, the eigenvalue problem with outgoing wave boundary conditions is addressed. With these conditions the system of equations can be nontrivially satisfied if

$$
m_{11}(\omega)=0 .
$$

Analytic continuation of the transfer matrix into the complex plane enables us to find solutions of (20) as complex eigenvalues $\omega .{ }^{24}$ By substituting the eigenvalue into the field representation (16) we obtain the corresponding eigenfunction, up to a complex constant. To solve (20) we apply a numerical iteration procedure of Newton type. ${ }^{25}$ In cases when that method fails to converge due to closely spaced eigenvalues, we use a more powerful technique for determining complex solutions, based on the argument principle method from complex analysis. ${ }^{26}$ 


\section{APPENDIX B. VARIATIONAL QNM MODEL OF THE TRANSMISSION PROBLEM}

We specialize to finite periodic structures that possess transmission properties with a bandgap, i.e. with a region of frequencies of very low transmission. Breaking the periodicity of the structure can give rise to defect resonances inside the bandgap. Approximation of the spectral transmission and of the associated field profiles for these resonances is the aim of our analysis. Therefore, we choose a field template for the transmittance problem as

$$
E(x, \omega) \simeq E_{m f}(x, \omega)+\sum_{p=1}^{N} a_{p}(\omega) Q_{p}(x),
$$

where $p$ is an index counting $N$ relevant QNMs, i.e. those with the real part of their complex frequency in the given frequency range. We showed in terms of the successful application of the template (21), that the transmission resonances associated with the defects are excited by the "mirror" field $E_{m f}$ of the periodic structure without defect, which for frequencies inside the bandgap is an almost completely reflected wave with only a weak tail that extends into the interior of the structure. Therefore, this template (21) quantifies the notion of a forced resonance response that appears because the incident wave possesses a real frequency close to the real part of the complex eigenfrequency of a suitable QNM supported by the defect structure.

This is only an approximate model for the transmittance problem in specific frequency regions, since neither $E_{m f}$ nor $Q$ satisfy all of equations (1)-(2). The residuals can be viewed as contributions from other QNMs in the complete set supported by the defect structure, that are not included in (21). To find the decomposition coefficients $a_{p}$, we use a variational form of the transmittance problem. ${ }^{18}$ The transmittance problem corresponds to the equation and natural boundary conditions, arising from the condition of stationarity of the functional

$$
\mathcal{L}(E)=\frac{1}{2} \int_{L}^{R}\left(\left(\partial_{x} E\right)^{2}-\frac{\omega^{2}}{c^{2}} n^{2}(x) E^{2}\right) d x-\frac{i \omega}{2 c}\left(\left.n_{\text {in }} E^{2}\right|_{x=L}+\left.n_{\text {out }} E^{2}\right|_{x=R}\right)+\left.2 i \frac{\omega}{c} n_{\text {in }} A_{\text {inc }} E\right|_{x=L} .
$$

If $\mathcal{L}$ becomes stationary, i.e. if the first variation of $\mathcal{L}(E)$ vanishes for arbitrary variations of $E$, then $E$ satisfies (1), and (2) as natural boundary conditions. Restricting the functional (22) to the field template $(21), \mathcal{L}$ becomes a function of the coefficients $a_{p}$, for given $E_{m f}$ and $Q_{p}$. The stationarity conditions then read

$$
\frac{\partial L}{\partial a_{q}}\left(a_{1}, a_{2}, \ldots, a_{N}\right)=0, \quad q=1, \ldots, N
$$

The optimal decomposition coefficients are obtained as solutions of a linear system

$$
\mathbf{A} \mathbf{a}=-\mathbf{b}
$$

where $\mathbf{a}=\left[a_{1}, a_{2}, \ldots, a_{N}\right]^{T}$ is the vector of coefficients to be determined by solving the system of equations (24). A and b are calculated according to $(21,22,23)$; explicit expressions are given in. ${ }^{19}$ For given frequency $\omega$ one thus approximates the field profile for a transmission problem with a specific incoming wave. Spectral information (transmittance, reflectance) can be obtained by repeating this procedure for a series of frequencies. The transmittance reads

$$
T(\omega)=\frac{1}{\left|A_{\text {inc }}\right|^{2}} \frac{n_{\text {out }}}{n_{\text {in }}}\left|E_{m f}(R, \omega)+\sum_{p=1}^{N} a_{p}(\omega) Q_{p}(R)\right|^{2} .
$$

We showed in ${ }^{19}$ that the mirror field is necessary for approximating the incoming part of the transmission field on the whole spatial region occupied by the structure. However, an additional approximation that is analytical in form can be obtained without the mirror field when only the spectral transmittance profile is considered. In cases where the underlying periodic sequence forms a good mirror, i.e. provides a high reflectance over the bandgap region, the mirror field could be omitted from the field template. This is possible because the mirror field contribution in the relevant terms of (24) becomes negligible for the field at the end of the structure where only outgoing waves are present. Then this approach can be seen as an alternative projection technique for a QNM expansion. 
Acknowledgment This work is financially supported by NanoNed, flagship NanoPhotonics, project TOE.7143.

\section{REFERENCES}

1. J.-M. Lourtioz, Photonic Crystals: Towards Nanoscale Photonic Devices, Springer, 2005.

2. K. Sakoda, Optical Properties Of Photonic Crystals, Springer, 2004.

3. C. K. Madsen and J. H. Zhao, Optical filter design and analysis: A signal processong approach, John Willey Sons, 1999.

4. P. Yeh, Optical waves in layered media, Willey, 1988.

5. E. S. C. Ching, P. T. Leung, A. M. van den Brink, W. M. Suen, S. S. Tong, and K. Young., "Quasi-normal mode expansion for waves in open systems," Rev. Mod. Phys 70(4), pp. 1545-1554, 1998.

6. A. Settimi, S. Severini, N. Mattiucci, C. Sibilia, M. Centini, G. D’ Aguanno, M. Bertolotti, M. Scalora, M. Bloemer, and C. M. Bowden, "Quasi-normal mode description of waves in one-dimensional photonic crystals," Phys. Rev E 68, pp. 026614/1-026614/11, 2003.

7. M. Bertolotti, "Linear one-dimensional resonant cavities," in Microresonators as building blocks for VLSI photonics, F.Michelotti, A.Driessen, and M.Bertolotti, eds., AIP Conference Proceedings 709, pp. 19-47, AIP, 2004.

8. B. Hoenders and M. Bertolotti, "The quasi normal mode description of the scattering process by dispersive photonic crystals," Proc. SPIE 6182, pp. 61821F1-9, 2006.

9. D. Felbacq and R. Smaâli, "Density of states for finite photonic crystals," Phys. Rev. B 67, p. 085105, 2003.

10. M. Bayindir, C. Kural, and E. Ozbay, "Coupled optical microcavities in one-dimensional photonic bandgap structures," J. Opt. A 3, pp. 184-189, 2001.

11. S. Lan, S. Nishikawa, Y. Sugimoto, N. Ikeda, K. Asakawa, and H. Ishikawa, "Analysis of defect coupling in one- and two-dimensional photonic crystals," Phys. Rev. B 65(16), p. 165208, 2002.

12. T. Kamalakis and T. Sphicopoulos, "Analytical expressions for the resonant frequencies and modal fields of finite coupled optical cavity chains,” IEEE J.Quant. El. 41(1), pp. 1419 - 1425, 2005.

13. A. Reynolds, U. Peschel, F. Lederer, P. Roberts, T. Krauss, and P. de Maagt, "Coupled defects in photonic crystals," IEEE Trans. MTT 49(10), pp. 1860 - 1867, 2001.

14. C. Vassallo, Optical Waveguide Concepts, Elsevier, Amsterdam, 1991.

15. H. Haus and W. Huang, "Coupled-mode theory," Proc. IEEE 79(10), pp. 1505-1518, 1991.

16. D. G. Hall and B. J. Thompson, eds., Selected Papers on Coupled-Mode Theory in Guided-Wave Optics, vol. MS 84 of SPIE Milestone Series, SPIE Optical Engineering Press, Bellingham, Washington USA, 1993.

17. W. P. Huang, "Coupled mode theory for optical waveguides: an overview," Journal of the Optical Society of America A 11(3), pp. 963-983, 1994.

18. E. W. C. van Groesen and J. Molenaar, Advanced Modeling in Science, SIAM publishers, 2007.

19. M. Maksimovic, M. Hammer, and E. van Groesen, "Field representation for optical defect resonances in multilayer microcavities using quasi-normal modes," Optics Comm. , 2007-accepted.

20. A. Sopahaluwakan, Characterization and simulation of localized states in periodic strucures, PhD Thesis, University of Twente, 2006.

21. F. Tisseur and K. Meerbergen, "The quadratic eigenvalue problem," SIAM Rev. 43(2), pp. 235-286, 2001.

22. S. Severini, A. Settimi, C. Sibilia, M. Bertolotti, A. Napoli, and A. Messina, "Second quantization and atomic spontaneous emission inside one-dimensional photonic crystals via a quasi-normal mode approach," Phys. Rev. E 70, pp. 056614-1-11, 2004.

23. M. Hammer, "Hybrid analytical/numerical coupled-mode modeling of guided wave devices," J. of Light. Tech. 25(9), pp. 2287-2298, 2007.

24. R. G. Newton, Scattering theory of waves and particles, Dover publications, 2002.

25. J. Mathews and K. Fink, Numerical Methods Using MATLAB, Prentice Hall, 1999.

26. D. Felbacq, "Numerical computation of resonance poles in scattering theory," Phys. Rev. E 64, p. 047702, 2001. 